\title{
Stability Criteria for Uncertain Discrete-Time Systems under the Influence of Saturation Nonlinearities and Time-Varying Delay
}

\author{
Siva Kumar Tadepalli, V. Krishna Rao Kandanvli, and Haranath Kar \\ Department of Electronics and Communication Engineering, Motilal Nehru National Institute of Technology Allahabad, \\ Allahabad 211004, India
}

Correspondence should be addressed to Siva Kumar Tadepalli; siva.kumar.1678@gmail.com

Received 23 January 2014; Accepted 12 February 2014; Published 1 April 2014

Academic Editors: F. Ding, C.-Y. Lu, and Q. Song

Copyright (C) 2014 Siva Kumar Tadepalli et al. This is an open access article distributed under the Creative Commons Attribution License, which permits unrestricted use, distribution, and reproduction in any medium, provided the original work is properly cited.

\begin{abstract}
The problem of global asymptotic stability of a class of uncertain discrete-time systems in the presence of saturation nonlinearities and interval-like time-varying delay in the state is considered. The uncertainties associated with the system parameters are assumed to be deterministic and normbounded. The objective of the paper is to propose stability criteria having considerably smaller numerical complexity. Two new delay-dependent stability criteria are derived by estimating the forward difference of the Lyapunov functional using the concept of reciprocal convexity and method of scale inequality, respectively. The presented criteria are compared with a previously reported criterion. A numerical example is provided to illustrate the effectiveness of the presented criteria.
\end{abstract}

\section{Introduction}

During the implementation of fixed-point state-space discrete-time systems using computer or digital hardware, one encounters finite wordlength nonlinearities such as quantization and overflow. Such nonlinearities may lead to instability in the designed system $[1,2]$. Saturation overflow nonlinearity is one of the well-known nonlinear phenomena studied in the real world [3]. The stability analysis of discretetime systems with state saturation is considered to be an important subject of system theoretic study [1-19].

Physical systems may suffer from parameter uncertainties that arise due to modeling errors, variations in system parameters, or some ignored factors. The existence of parameter uncertainties may result in instability of the designed system [20].

In the modeling of physical systems, time delays are often introduced due to finite capabilities of information processing and data transmission among various parts of the system $[17,20-22]$. Such delays are another source of instability in discrete-time systems. The stability criteria for time delay systems are broadly classified into delay-independent and delay-dependent. In general, delay-dependent approach [15, $17,18,20,22-37]$ leads to less conservative results as compared to delay-independent approach $[16,19,20,38]$. The delay partitioning approach has been utilized in $[30,31]$ for the stability analysis of systems with interval-like time-varying delay.

The stability analysis of discrete-time systems involving overflow nonlinearities, parameter uncertainties, and state delays is an important problem. Delay-independent stability criteria for a class of discrete-time state-delayed systems with saturation nonlinearities have been presented in [16, 19]. A delay-dependent approach for the stability analysis of uncertain discrete-time systems with time-varying delays and quantization/overflow nonlinearities has been proposed in [17]. In a recent work [18], a delay-dependent global asymptotic stability criterion for a class of uncertain discretetime state-delayed systems with saturation nonlinearities has been established. 
A major concern of the delay-dependent stability criteria is computational complexity. The objective of this paper is to present global asymptotic stability criteria for uncertain discrete-time systems under the influence of saturation nonlinearities and time-varying delay. In particular, inspired by $[23,29,39]$, we are interested to develop delay-dependent stability criteria which are numerically less complex as compared to [18].

The paper is organized as follows. Section 2 defines the system under consideration and presents a recently reported criterion. In Section 3, we specify the lemmas used and then present our results. A numerical example illustrating the usefulness of the presented results is given in Section 4 .

Notations. The notations used throughout this paper are standard. $\mathbb{R}^{p}$ denotes the $p$-dimensional Euclidean space; $\mathbb{R}^{p \times q}$ is the set of $p \times q$ real matrices; 0 represents null matrix or null vector of appropriate dimension; $\mathbf{I}$ is the identity matrix of appropriate dimension; $\mathbf{B}^{T}$ stands for the transpose of the matrix (or vector) $\mathbf{B} ; \mathbf{B}>\mathbf{0}(\geq \mathbf{0})$ means that $\mathbf{B}$ is positive definite (semidefinite) symmetric matrix; $\mathbf{B}<\mathbf{0}$ represents that $\mathbf{B}$ is negative definite symmetric matrix; $\lfloor m\rceil$ denotes a function which returns the nearest integer to $m ;|m|$ stands for absolute value of a real number $m$; the symbol $*$ represents the symmetric terms in a symmetric matrix.

\section{System Description and Existing Criterion}

The system under consideration is given by

$$
\begin{aligned}
\mathbf{x}(k+1) & =\mathbf{f}(\mathbf{y}(k)) \\
& =\left[\begin{array}{llll}
f_{1}\left(y_{1}(k)\right) & f_{2}\left(y_{2}(k)\right) & \cdots & f_{n}\left(y_{n}(k)\right)
\end{array}\right]^{T} \\
\mathbf{y}(k) & =\left(\begin{array}{llll}
\mathbf{A}+\Delta \mathbf{A}) \mathbf{x}(k)+\left(\mathbf{A}_{d}+\Delta \mathbf{A}_{d}\right) \mathbf{x}(k-d(k)
\end{array}\right) \\
& =\left[\begin{array}{llll}
y_{1}(k) & y_{2}(k) & \cdots & y_{n}(k)
\end{array}\right]^{T} \\
\mathbf{x}(k) & =\boldsymbol{\varphi}(k), \quad \forall k=-h_{2},-h_{2}+1, \ldots, 0,
\end{aligned}
$$

where $\mathbf{x}(k) \in \mathbb{R}^{n}$ is the system state vector; $\mathbf{A}, \mathbf{A}_{d} \in \mathbb{R}^{n \times n}$ are the known constant matrices; $\Delta \mathbf{A}, \Delta \mathbf{A}_{d} \in \mathbb{R}^{n \times n}$ are the unknown matrices representing parametric uncertainties in the state matrices; $\varphi(k) \in \mathbb{R}^{n}$ is the initial condition at $k$; and the time-varying delay $d(k)$ is a positive integer which satisfies

$$
h_{1} \leq d(k) \leq h_{2}
$$

where $h_{1}$ and $h_{2}$ are known nonnegative integers representing the lower and upper delay bounds, respectively.

The saturation nonlinearities given by

$$
f_{i}\left(y_{i}(k)\right)= \begin{cases}y_{i}(k), & \left|y_{i}(k)\right| \leq 1 \\ 1, & y_{i}(k)>1 \\ -1, & y_{i}(k)<-1, \\ & i=1,2, \ldots, n\end{cases}
$$

are under consideration. The uncertainties are assumed to be of the form $[16,17,25,38,40]$

$$
\begin{aligned}
\Delta \mathbf{A} & =\mathbf{H}_{0} \mathbf{F}_{0} \mathbf{E}_{0}, \\
\Delta \mathbf{A}_{d} & =\mathbf{H}_{1} \mathbf{F}_{1} \mathbf{E}_{1},
\end{aligned}
$$

where $\mathbf{H}_{i} \in \mathbb{R}^{n \times p_{i}}$ and $\mathbf{E}_{i} \in \mathbb{R}^{q_{i} \times n}(i=0,1)$ are known constant matrices and $\mathbf{F}_{i} \in \mathbb{R}^{p_{i} \times q_{i}}(i=0,1)$ is an unknown matrix which satisfies

$$
\mathbf{F}_{i}^{T} \mathbf{F}_{i} \leq \mathbf{I}, \quad i=0,1 .
$$

Pertaining to the system given by (1)-(8), the following criterion has been recently reported in [18].

Theorem $\mathbf{1}$ (see [18]). Suppose $\mathbf{C}=\left[c_{i j}\right] \in \mathbb{R}^{n \times n}$ denotes $a$ matrix defined by

$$
\begin{aligned}
& c_{i i}=\sum_{j=1, j \neq i}^{n}\left(\alpha_{i j}+\beta_{i j}\right), \quad i=1,2, \ldots, n, \\
& c_{i j}=\alpha_{i j}-\beta_{i j}, \quad i, j=1,2, \ldots, n(i \neq j), \\
& \alpha_{i j}>0, \quad \beta_{i j}>0, \quad i, j=1,2, \ldots, n(i \neq j),
\end{aligned}
$$

where for $n=1, \mathbf{C}$ corresponds to a scalar $\mu>0$. Then, for given positive integers $h_{1}$ and $h_{2}$ with $h_{2} \geq h_{1}$, the system described by (1)-(8) is globally asymptotically stable if there exist appropriately dimensioned matrices $\mathbf{P}>\mathbf{0}, \mathbf{Q}_{i}>\mathbf{0}(i=$ $1,2,3), \mathbf{R}_{1}>\mathbf{0}, \mathbf{R}_{2}>\mathbf{0}, \mathbf{X}=\left[\begin{array}{cc}\mathbf{X}_{11} & \mathbf{X}_{12} \\ * & \mathbf{X}_{22}\end{array}\right] \geq \mathbf{0}, \mathbf{Y}=\left[\begin{array}{cc}\mathbf{Y}_{11} & \mathbf{Y}_{12} \\ * & \mathbf{Y}_{22}\end{array}\right] \geq \mathbf{0}$, $\mathbf{N}=\left[\begin{array}{l}\mathbf{N}_{1} \\ \mathbf{N}_{2}\end{array}\right], \mathbf{M}=\left[\begin{array}{l}\mathbf{M}_{1} \\ \mathbf{M}_{2}\end{array}\right]$, and $\mathbf{W}=\left[\begin{array}{l}\mathbf{W}_{1} \\ \mathbf{w}_{2}\end{array}\right]$ and positive scalars $\alpha_{i j}$, $\beta_{i j}(i, j=1,2, \ldots, n(i \neq j)), \epsilon_{0}$, and $\epsilon_{1}$ such that the following linear matrix inequalities (LMIs) hold:

$$
\begin{gathered}
{\left[\begin{array}{ccccccc}
\boldsymbol{\xi}_{11} & \boldsymbol{\xi}_{12} & \mathbf{W}_{1} & -\mathbf{M}_{1} & \mathbf{A}^{T} \mathbf{C}-\overline{\mathbf{R}} & \mathbf{0} & \mathbf{0} \\
* & \boldsymbol{\xi}_{22} & \mathbf{W}_{2} & -\mathbf{M}_{2} & \mathbf{A}_{d}^{T} \mathbf{C} & \mathbf{0} & \mathbf{0} \\
* & * & -\mathbf{Q}_{1} & \mathbf{0} & \mathbf{0} & \mathbf{0} & \mathbf{0} \\
* & * & * & -\mathbf{Q}_{2} & \mathbf{0} & \mathbf{0} & \mathbf{0} \\
* & * & * & * & \boldsymbol{\xi}_{55} & \mathbf{C}^{T} \mathbf{H}_{0} & \mathbf{C}^{T} \mathbf{H}_{1} \\
* & * & * & * & * & -\boldsymbol{\epsilon}_{0} \mathbf{I} & \mathbf{0} \\
* & * & * & * & * & * & -\boldsymbol{\epsilon}_{1} \mathbf{I}
\end{array}\right]<\mathbf{0}} \\
\\
{\left[\begin{array}{cc}
\mathbf{X} & \mathbf{N} \\
* & \mathbf{R}_{1}
\end{array}\right] \geq \mathbf{0}} \\
{\left[\begin{array}{cc}
\mathbf{Y} & \mathbf{W} \\
* & \mathbf{R}_{2}
\end{array}\right] \geq \mathbf{0}} \\
\left.\begin{array}{cc}
\mathbf{X}+\mathbf{Y} & \mathbf{M} \\
* & \mathbf{R}_{1}+\mathbf{R}_{2}
\end{array}\right] \geq \mathbf{0}
\end{gathered}
$$


where

$$
\begin{aligned}
\overline{\mathbf{R}}= & h_{2} \mathbf{R}_{1}+h_{12} \mathbf{R}_{2}, \\
\xi_{11}= & -\mathbf{P}+\mathbf{Q}_{1}+\mathbf{Q}_{2}+\left(h_{12}+1\right) \mathbf{Q}_{3}+\overline{\mathbf{R}} \\
& +\mathbf{N}_{1}+\mathbf{N}_{1}^{T}+h_{2} \mathbf{X}_{11}+h_{12} \mathbf{Y}_{11}+\epsilon_{0} \mathbf{E}_{0}^{T} \mathbf{E}_{0}, \\
\xi_{12}= & -\mathbf{N}_{1}+\mathbf{N}_{2}^{T}+\mathbf{M}_{1}-\mathbf{W}_{1}+h_{2} \mathbf{X}_{12} \\
& +h_{12} \mathbf{Y}_{12}, \\
\xi_{22}= & -\mathbf{N}_{2}-\mathbf{N}_{2}^{T}+\mathbf{M}_{2}+\mathbf{M}_{2}^{T}-\mathbf{W}_{2}-\mathbf{W}_{2}^{T} \\
& -\mathbf{Q}_{3}+h_{2} \mathbf{X}_{22}+h_{12} \mathbf{Y}_{22}+\epsilon_{1} \mathbf{E}_{1}^{T} \mathbf{E}_{1}, \\
\boldsymbol{\xi}_{55}= & \mathbf{P}-\left(\mathbf{C}+\mathbf{C}^{T}\right)+\overline{\mathbf{R}}, \\
h_{12}= & h_{2}-h_{1}
\end{aligned}
$$

Remark 2. For $\mathbf{A} \in \mathbb{R}^{n \times n}$ and $\mathbf{H}_{i} \in \mathbb{R}^{n \times p}(i=0,1)$, the total row size of the LMIs in Theorem 1 is $L_{1}=2 n^{2}+22 n+2 p+2$ and the total number of scalar decision variables is $M_{1}=15 n^{2}+$ $3 n+2$. The numerical complexity of Theorem 1 is proportional to $L_{1}\left(M_{1}\right)^{3}[26]$.

\section{Main Results}

Before presenting the main results of the paper, we recall the following lemmas.

Lemma 3 (see $[29,39]$ ). For any vectors $\boldsymbol{\xi}_{1}$ and $\boldsymbol{\xi}_{2}$, matrices $\mathbf{R}$ and $\mathbf{S}$, and real numbers $\alpha_{1} \geq 0$ and $\alpha_{2} \geq 0$ satisfying

$$
\left[\begin{array}{ll}
\mathbf{R} & \mathbf{S} \\
* & \mathbf{R}
\end{array}\right] \geq \mathbf{0}, \quad \alpha_{1}+\alpha_{2}=1,
$$

$\boldsymbol{\xi}_{i}=\mathbf{0}$, if $\alpha_{i}=0, i=1,2$, then

$$
-\frac{1}{\alpha_{1}} \boldsymbol{\xi}_{1}{ }^{T} \mathbf{R} \boldsymbol{\xi}_{1}-\frac{1}{\alpha_{2}} \boldsymbol{\xi}_{2}{ }^{T} \mathbf{R} \boldsymbol{\xi}_{2} \leq-\left[\begin{array}{l}
\boldsymbol{\xi}_{1} \\
\boldsymbol{\xi}_{2}
\end{array}\right]^{T}\left[\begin{array}{ll}
\mathbf{R} & \mathbf{S} \\
* & \mathbf{R}
\end{array}\right]\left[\begin{array}{l}
\boldsymbol{\xi}_{1} \\
\boldsymbol{\xi}_{2}
\end{array}\right] .
$$

Lemma 4 (see $[40,41]$ ). Let $\Sigma, \Gamma, \mathbf{F}$, and $\Lambda$ be real matrices of appropriate dimensions with $\boldsymbol{\Lambda}$ satisfying $\Lambda=\Lambda^{T}$; then,

$$
\boldsymbol{\Lambda}+\boldsymbol{\Sigma} \mathbf{F} \boldsymbol{\Gamma}+\boldsymbol{\Gamma}^{T} \mathbf{F}^{T} \boldsymbol{\Sigma}^{T}<\mathbf{0}
$$

for all $\mathbf{F}^{T} \mathbf{F} \leq \mathbf{I}$, if and only if there exists a scalar $\epsilon>0$ such that

$$
\boldsymbol{\Lambda}+\epsilon^{-1} \boldsymbol{\Sigma} \boldsymbol{\Sigma}^{T}+\epsilon \boldsymbol{\Gamma}^{T} \boldsymbol{\Gamma}<\mathbf{0} .
$$

Lemma 5 (see [28]). For any positive definite matrix $\mathbf{J} \in \mathbb{R}^{n \times n}$, two positive integers $r$ and $r_{0}$ satisfying $r \geq r_{0} \geq 1$, and vector function $\mathbf{x}(i) \in \mathbb{R}^{n}$, one has

$$
\left(\sum_{i=r_{0}}^{r} \mathbf{x}(i)\right)^{T} \mathbf{J}\left(\sum_{i=r_{0}}^{r} \mathbf{x}(i)\right) \leq\left(r-r_{0}+1\right) \sum_{i=r_{0}}^{r} \mathbf{x}^{T}(i) \mathbf{J} \mathbf{x}(i) .
$$

In the following, inspired by the work of $[12,14,23,29$, 39], we will establish delay-dependent criteria for the global asymptotic stability of the system given by (1)-(8).
Theorem 6. Given two integers $h_{1}$ and $h_{2}$ satisfying $0 \leq h_{1} \leq$ $h_{2}$, the system represented by (1)-(8) is globally asymptotically stable if there exist matrices $\mathbf{P}>\mathbf{0}, \mathbf{Q}_{i}>\mathbf{0}(i=1,2,3), \mathbf{R}_{1}>$ $\mathbf{0}$, and $\mathbf{R}_{2}>\mathbf{0}$; a matrix $\mathbf{S}$ with compatible dimensions; and positive scalars $\epsilon_{0}, \epsilon_{1}, \alpha_{i j}$, and $\beta_{i j}(i, j=1,2, \ldots, n(i \neq j))$ such that

$$
\begin{aligned}
\boldsymbol{\Theta}= & {\left[\begin{array}{cc}
\mathbf{R}_{2} & \mathbf{S} \\
* & \mathbf{R}_{2}
\end{array}\right]>\mathbf{0}, } \\
\Psi= & \mathbf{v}_{1}^{T}\left[-\mathbf{P}+\sum_{i=1}^{3} \mathbf{Q}_{i}+h_{12} \mathbf{Q}_{3}+\epsilon_{0} \mathbf{E}_{0}^{T} \mathbf{E}_{0}\right] \mathbf{v}_{1} \\
& +\left(\mathbf{v}_{1}-\mathbf{v}_{5}\right)^{T}\left(h_{1}^{2} \mathbf{R}_{1}+h_{12}^{2} \mathbf{R}_{2}\right)\left(\mathbf{v}_{1}-\mathbf{v}_{5}\right) \\
& +\mathbf{v}_{2}^{T} \mathbf{A}_{d}^{T} \mathbf{C} \mathbf{v}_{5}-\left(\mathbf{v}_{1}-\mathbf{v}_{3}\right)^{T} \mathbf{R}_{1}\left(\mathbf{v}_{1}-\mathbf{v}_{3}\right) \\
& -\left(\mathbf{v}_{2}-\mathbf{v}_{4}\right)^{T} \mathbf{R}_{2}\left(\mathbf{v}_{2}-\mathbf{v}_{4}\right)-\mathbf{v}_{4}^{T} \mathbf{S}^{T} \mathbf{v}_{2}+\mathbf{v}_{4}^{T} \mathbf{S}^{T} \mathbf{v}_{3} \\
& +\mathbf{v}_{2}^{T}\left[\mathbf{S}+\mathbf{S}^{T}-\mathbf{Q}_{3}+\epsilon_{1} \mathbf{E}_{1}^{T} \mathbf{E}_{1}\right] \mathbf{v}_{2}-\mathbf{v}_{2}^{T} \mathbf{S}^{T} \mathbf{v}_{3} \\
& -\left(\mathbf{v}_{2}-\mathbf{v}_{3}\right)^{T} \mathbf{R}_{2}\left(\mathbf{v}_{2}-\mathbf{v}_{3}\right)-\mathbf{v}_{2}^{T} \mathbf{S}_{4}-\mathbf{v}_{3}^{T} \mathbf{S}_{2} \\
& +\mathbf{v}_{3}^{T} \mathbf{S} \mathbf{v}_{4}-\mathbf{v}_{3}^{T} \mathbf{Q}_{1} \mathbf{v}_{3}-\mathbf{v}_{4}^{T} \mathbf{Q}_{2} \mathbf{v}_{4}+\mathbf{v}_{1}^{T} \mathbf{A}^{T} \mathbf{C} \mathbf{v}_{5} \\
& +\mathbf{v}_{5}^{T} \mathbf{C}^{T} \mathbf{A} \mathbf{v}_{1}+\mathbf{v}_{5}^{T} \mathbf{C}^{T} \mathbf{A}_{d} \mathbf{v}_{2}+\mathbf{v}_{5}^{T} \mathbf{C}^{T} \mathbf{H}_{0} \mathbf{v}_{6} \\
& +\mathbf{v}_{5}^{T}\left[\mathbf{P}-\left(\mathbf{C}+\mathbf{C}^{T}\right)\right] \mathbf{v}_{5}+\mathbf{v}_{6}^{T} \mathbf{H}_{0}^{T} \mathbf{C} \mathbf{v}_{5} \\
& +\mathbf{v}_{5}^{T} \mathbf{C}^{T} \mathbf{H}_{1} \mathbf{v}_{7}+\mathbf{v}_{7}^{T} \mathbf{H}_{1}^{T} \mathbf{C} \mathbf{v}_{5}-\epsilon_{0} \mathbf{v}_{6}^{T} \mathbf{v}_{6} \\
& -\epsilon_{1} \mathbf{v}_{7}^{T} \mathbf{I} \mathbf{v}_{7}<\mathbf{0},
\end{aligned}
$$

where $\mathbf{C}$ is defined by (9)-(11) and $\mathbf{v}_{i}(i=1,2, \ldots, 7)$ denotes the block entry matrix, for example, $\mathbf{v}_{4}=\left[\begin{array}{lllllll}\mathbf{0} & \mathbf{0} & \mathbf{0} & \mathbf{I} & \mathbf{0} & \mathbf{0} & \mathbf{0}\end{array}\right]$.

Proof. Consider the following Lyapunov functional candidate [23]:

$$
\begin{aligned}
V(\mathbf{x}(k))= & \mathbf{x}^{T}(k) \mathbf{P} \mathbf{x}(k)+\sum_{i=k-h_{1}}^{k-1} \mathbf{x}^{T}(i) \mathbf{Q}_{1} \mathbf{x}(i) \\
& +\sum_{i=k-h_{2}}^{k-1} \mathbf{x}^{T}(i) \mathbf{Q}_{2} \mathbf{x}(i) \\
& +\sum_{j=-h_{2}}^{-h_{1}} \sum_{i=k+j}^{k-1} \mathbf{x}^{T}(i) \mathbf{Q}_{3} \mathbf{x}(i) \\
& +h_{1} \sum_{\theta=-h_{1}+1}^{0} \sum_{j=k-1+\theta}^{k-1} \boldsymbol{\eta}^{T}(j) \mathbf{R}_{1} \boldsymbol{\eta}(j) \\
& +h_{12} \sum_{\theta=-h_{2}+1}^{-h_{1}} \sum_{j=k-1+\theta}^{k-1} \boldsymbol{\eta}^{T}(j) \mathbf{R}_{2} \boldsymbol{\eta}(j),
\end{aligned}
$$

where

$$
\boldsymbol{\eta}(k)=\mathbf{x}(k+1)-\mathbf{x}(k)=\mathbf{f}(\mathbf{y}(k))-\mathbf{x}(k) .
$$


Taking the forward difference of the Lyapunov functional (21) along the solutions of (1) yields

$$
\begin{aligned}
\Delta V(\mathbf{x}(k))= & V(\mathbf{x}(k+1))-V(\mathbf{x}(k)) \\
= & \mathbf{f}^{T}(\mathbf{y}(k)) \mathbf{P} \mathbf{f}(\mathbf{y}(k))-\mathbf{x}^{T}(k) \mathbf{P} \mathbf{x}(k) \\
& +\mathbf{x}^{T}(k) \mathbf{Q}_{1} \mathbf{x}(k)+\mathbf{x}^{T}(k) \mathbf{Q}_{2} \mathbf{x}(k) \\
& -\mathbf{x}^{T}\left(k-h_{1}\right) \mathbf{Q}_{1} \mathbf{x}\left(k-h_{1}\right) \\
& -\mathbf{x}^{T}\left(k-h_{2}\right) \mathbf{Q}_{2} \mathbf{x}\left(k-h_{2}\right) \\
& +\left(h_{12}+1\right) \mathbf{x}^{T}(k) \mathbf{Q}_{3} \mathbf{x}(k) \\
& -\sum_{i=k-h_{2}}^{k-h_{1}} \mathbf{x}^{T}(i) \mathbf{Q}_{3} \mathbf{x}(i)+h_{1}^{2} \boldsymbol{\eta}^{T}(k) \mathbf{R}_{1} \boldsymbol{\eta}(k) \\
& +h_{12}^{2} \boldsymbol{\eta}^{T}(k) \mathbf{R}_{2} \boldsymbol{\eta}(k) \\
& -h_{1} \sum_{i=k-h_{1}}^{k-1} \boldsymbol{\eta}^{T}(i) \mathbf{R}_{1} \boldsymbol{\eta}(i) \\
& -h_{12} \sum_{i=k-h_{1}-1} \boldsymbol{\eta}^{T}(i) \mathbf{R}_{2} \boldsymbol{\eta}(i) .
\end{aligned}
$$

Using Lemmas 3 and 5, one can obtain the following relation if there exists a matrix $\mathbf{S}$ such that (19) holds [29]:

$$
\begin{aligned}
-h_{12} \sum_{i=k-h_{2}}^{k-h_{1}-1} \boldsymbol{\eta}^{T}(i) \mathbf{R}_{2} \boldsymbol{\eta}(i) \\
\leq-\frac{1}{\left(d(k)-h_{1}\right) / h_{12}} \gamma_{1}^{T}(k) \mathbf{R}_{2} \boldsymbol{\gamma}_{1}(k) \\
-\frac{1}{\left(h_{2}-d(k)\right) / h_{12}} \gamma_{2}^{T}(k) \mathbf{R}_{2} \boldsymbol{\gamma}_{2}(k) \\
\leq-\left[\begin{array}{l}
\gamma_{1}(k) \\
\gamma_{2}(k)
\end{array}\right]^{T} \Theta\left[\begin{array}{l}
\gamma_{1}(k) \\
\gamma_{2}(k)
\end{array}\right],
\end{aligned}
$$

where

$$
\begin{aligned}
& \gamma_{1}(k)=\mathbf{x}\left(k-h_{1}\right)-\mathbf{x}(k-d(k)), \\
& \gamma_{2}(k)=\mathbf{x}(k-d(k))-\mathbf{x}\left(k-h_{2}\right) .
\end{aligned}
$$

It follows from Lemma 5 that [29]

$$
\begin{aligned}
& -h_{1} \sum_{i=k-h_{1}}^{k-1} \boldsymbol{\eta}^{T}(i) \mathbf{R}_{1} \boldsymbol{\eta}(i) \\
& \quad \leq-\sum_{i=k-h_{1}}^{k-1} \boldsymbol{\eta}^{T}(i) \mathbf{R}_{1} \sum_{i=k-h_{1}}^{k-1} \boldsymbol{\eta}(i) \\
& \quad=-\left[\mathbf{x}^{T}(k)-\mathbf{x}^{T}\left(k-h_{1}\right)\right] \mathbf{R}_{1}\left[\mathbf{x}(k)-\mathbf{x}\left(k-h_{1}\right)\right] .
\end{aligned}
$$

Note that

$-\sum_{i=k-h_{2}}^{k-h_{1}} \mathbf{x}^{T}(i) \mathbf{Q}_{3} \mathbf{x}(i) \leq-\mathbf{x}^{T}(k-d(k)) \mathbf{Q}_{3} \mathbf{x}(k-d(k))$.

Employing (23)-(28), we have the following inequality:

$$
\Delta V(\mathbf{x}(k)) \leq \boldsymbol{\xi}^{T}(k) \Psi_{1} \xi(k)-\delta,
$$

where

$$
\begin{aligned}
& \delta=\sum_{i=1}^{n} 2\left[y_{i}(k)-f_{i}\left(y_{i}(k)\right)\right] \\
& \times\left[\sum _ { j = 1 , j \neq i } ^ { n } \left\{\left(\alpha_{i j}+\beta_{i j}\right) f_{i}\left(y_{i}(k)\right)\right.\right. \\
& \left.\left.+\left(\alpha_{i j}-\beta_{i j}\right) f_{j}\left(y_{j}(k)\right)\right\}\right] \\
& =\mathbf{y}^{T}(k) \mathbf{C} \mathbf{f}(\mathbf{y}(k))+\mathbf{f}^{T}(\mathbf{y}(k)) \mathbf{C}^{T} \mathbf{y}(k) \\
& -\mathbf{f}^{T}(\mathbf{y}(k))\left(\mathbf{C}+\mathbf{C}^{T}\right) \mathbf{f}(\mathbf{y}(k)), \\
& \Psi_{1}=\mathbf{e}_{1}^{T}\left[-\mathbf{P}+\sum_{i=1}^{3} \mathbf{Q}_{i}+h_{12} \mathbf{Q}_{3}\right] \mathbf{e}_{1} \\
& +\left(\mathbf{e}_{1}-\mathbf{e}_{5}\right)^{T}\left(h_{1}^{2} \mathbf{R}_{1}+h_{12}^{2} \mathbf{R}_{2}\right)\left(\mathbf{e}_{1}-\mathbf{e}_{5}\right) \\
& +\mathbf{e}_{2}^{T} \overline{\mathbf{A}}_{d}^{T} \mathbf{C e}_{5}-\left(\mathbf{e}_{1}-\mathbf{e}_{3}\right)^{T} \mathbf{R}_{1}\left(\mathbf{e}_{1}-\mathbf{e}_{3}\right) \\
& -\left(\mathbf{e}_{2}-\mathbf{e}_{4}\right)^{T} \mathbf{R}_{2}\left(\mathbf{e}_{2}-\mathbf{e}_{4}\right)-\mathbf{e}_{4}^{T} \mathbf{S}^{T} \mathbf{e}_{2}+\mathbf{e}_{4}^{T} \mathbf{S}^{T} \mathbf{e}_{3} \\
& +\mathbf{e}_{2}^{T}\left[\mathbf{S}+\mathbf{S}^{T}-\mathbf{Q}_{3}\right] \mathbf{e}_{2}-\mathbf{e}_{2}^{T} \mathbf{S}^{T} \mathbf{e}_{3} \\
& -\left(\mathbf{e}_{2}-\mathbf{e}_{3}\right)^{T} \mathbf{R}_{2}\left(\mathbf{e}_{2}-\mathbf{e}_{3}\right)-\mathbf{e}_{2}^{T} \mathbf{S} \mathbf{e}_{4}-\mathbf{e}_{3}^{T} \mathbf{S} \mathbf{e}_{2} \\
& +\mathbf{e}_{3}^{T} \mathbf{S} \mathbf{e}_{4}-\mathbf{e}_{3}^{T} \mathbf{Q}_{1} \mathbf{e}_{3}-\mathbf{e}_{4}^{T} \mathbf{Q}_{2} \mathbf{e}_{4}+\mathbf{e}_{1}^{T} \bar{A}^{T} \mathbf{C} \mathbf{e}_{5} \\
& +\mathbf{e}_{5}^{T} \mathbf{C}^{T} \overline{\mathbf{A}} \mathbf{e}_{1}+\mathbf{e}_{5}^{T} \mathbf{C}^{T} \overline{\mathbf{A}}_{d} \mathbf{e}_{2} \\
& +\mathbf{e}_{5}^{T}\left[\mathbf{P}-\left(\mathbf{C}+\mathbf{C}^{T}\right)\right] \mathbf{e}_{5}, \\
& \overline{\mathbf{A}}=\mathbf{A}+\Delta \mathbf{A}, \quad \overline{\mathbf{A}}_{d}=\mathbf{A}_{d}+\Delta \mathbf{A}_{d}, \\
& \boldsymbol{\xi}(k)=\left[\begin{array}{lll}
\mathbf{x}^{T}(k) & \mathbf{x}^{T}(k-d(k)) & \mathbf{x}^{T}\left(k-h_{1}\right)
\end{array}\right. \\
& \left.\mathbf{x}^{T}\left(k-h_{2}\right) \mathbf{f}^{T}(\mathbf{y}(k))\right]^{T} .
\end{aligned}
$$

Here, $\mathbf{e}_{i}(i=1,2, \ldots, 5)$ is the block entry matrix, for example, $\mathbf{e}_{4}=\left[\begin{array}{lllll}\mathbf{0} & \mathbf{0} & \mathbf{0} & \text { I } & \mathbf{0}\end{array}\right]$. Observe that, for the saturation nonlinearities given by (5) along with (11), the quantity $\delta$ (see (30)) is nonnegative $[12,14]$. (It is understood that for $n=1, \delta$ is given by $\delta=2 \mu\left[y_{1}(k)-f_{1}\left(y_{1}(k)\right)\right] f_{1}\left(y_{1}(k)\right)$, where $\mu>0$. Clearly, in such case $\mathbf{C}$ reduces to a positive scalar, namely, $\mu$ [14]).

From (29), it is clear that $\Delta V(\mathbf{x}(k))<0$, if $\boldsymbol{\Psi}_{1}<\mathbf{0}$. Thus, $\Psi_{1}<\mathbf{0}$ and (19) are sufficient conditions for the global 
asymptotic stability of the system given by (1)-(8). Further, using (6), the condition $\Psi_{1}<\mathbf{0}$ can be rewritten in the following form:

$$
\boldsymbol{\Lambda}_{0}+\overline{\mathbf{H}}_{0} \mathbf{F}_{0} \overline{\mathbf{E}}_{0}+\overline{\mathbf{E}}_{0}^{T} \mathbf{F}_{0}^{T} \overline{\mathbf{H}}_{0}^{T}<\mathbf{0}
$$

where

$$
\begin{aligned}
& \Lambda_{0}=\mathbf{e}_{1}^{T}\left[-\mathbf{P}+\sum_{i=1}^{3} \mathbf{Q}_{i}+h_{12} \mathbf{Q}_{3}\right] \mathbf{e}_{1} \\
& +\left(\mathbf{e}_{1}-\mathbf{e}_{5}\right)^{T}\left(h_{1}^{2} \mathbf{R}_{1}+h_{12}^{2} \mathbf{R}_{2}\right)\left(\mathbf{e}_{1}-\mathbf{e}_{5}\right) \\
& +\mathbf{e}_{2}^{T} \overline{\mathbf{A}}_{d}^{T} \mathbf{C e}_{5}-\left(\mathbf{e}_{1}-\mathbf{e}_{3}\right)^{T} \mathbf{R}_{1}\left(\mathbf{e}_{1}-\mathbf{e}_{3}\right) \\
& -\left(\mathbf{e}_{2}-\mathbf{e}_{4}\right)^{T} \mathbf{R}_{2}\left(\mathbf{e}_{2}-\mathbf{e}_{4}\right)-\mathbf{e}_{4}^{T} \mathbf{S}^{T} \mathbf{e}_{2}+\mathbf{e}_{4}^{T} \mathbf{S}^{T} \mathbf{e}_{3} \\
& +\mathbf{e}_{2}^{T}\left[\mathbf{S}+\mathbf{S}^{T}-\mathbf{Q}_{3}\right] \mathbf{e}_{2}-\mathbf{e}_{2}^{T} \mathbf{S}^{T} \mathbf{e}_{3} \\
& -\left(\mathbf{e}_{2}-\mathbf{e}_{3}\right)^{T} \mathbf{R}_{2}\left(\mathbf{e}_{2}-\mathbf{e}_{3}\right)-\mathbf{e}_{2}^{T} \mathbf{S e}_{4}-\mathbf{e}_{3}^{T} \mathbf{S} \mathbf{e}_{2} \\
& +\mathbf{e}_{3}^{T} \mathbf{S} \mathbf{e}_{4}-\mathbf{e}_{3}^{T} \mathbf{Q}_{1} \mathbf{e}_{3}-\mathbf{e}_{4}^{T} \mathbf{Q}_{2} \mathbf{e}_{4}+\mathbf{e}_{1}^{T} \mathbf{A}^{T} \mathbf{C} \mathbf{e}_{5} \\
& +\mathbf{e}_{5}^{T} \mathbf{C}^{T} \mathbf{A e}_{1}+\mathbf{e}_{5}^{T} \mathbf{C}^{T} \overline{\mathbf{A}}_{d} \mathbf{e}_{2}+\mathbf{e}_{5}^{T}\left[\mathbf{P}-\left(\mathbf{C}+\mathbf{C}^{T}\right)\right] \mathbf{e}_{5}, \\
& \overline{\mathbf{H}}_{0}^{T}=\left[\begin{array}{lllll}
\mathbf{0} & \mathbf{0} & \mathbf{0} & \mathbf{0} & \mathbf{H}_{0}^{T} \mathbf{C}
\end{array}\right], \\
& \overline{\mathbf{E}}_{0}=\left[\begin{array}{lllll}
\mathbf{E}_{0} & \mathbf{0} & \mathbf{0} & \mathbf{0} & \mathbf{0}
\end{array}\right] \text {. }
\end{aligned}
$$

By Lemma 4, (34) is equivalent to

$$
\boldsymbol{\Lambda}_{0}+\epsilon_{0}^{-1} \overline{\mathbf{H}}_{0} \overline{\mathbf{H}}_{0}^{T}+\epsilon_{0} \overline{\mathbf{E}}_{0}^{T} \overline{\mathbf{E}}_{0}<\mathbf{0}
$$

where $\epsilon_{0}>0$. By employing the well-known Schur complement [41], (36) can also be expressed as

$$
\begin{aligned}
\mathbf{u}_{1}^{T}[ & \left.-\mathbf{P}+\sum_{i=1}^{3} \mathbf{Q}_{i}+h_{12} \mathbf{Q}_{3}+\epsilon_{0} \mathbf{E}_{0}^{T} \mathbf{E}_{0}\right] \mathbf{u}_{1} \\
& +\left(\mathbf{u}_{1}-\mathbf{u}_{5}\right)^{T}\left(h_{1}^{2} \mathbf{R}_{1}+h_{12}^{2} \mathbf{R}_{2}\right)\left(\mathbf{u}_{1}-\mathbf{u}_{5}\right) \\
& +\mathbf{u}_{2}^{T} \overline{\mathbf{A}}_{d}^{T} \mathbf{C} \mathbf{u}_{5}-\left(\mathbf{u}_{1}-\mathbf{u}_{3}\right)^{T} \mathbf{R}_{1}\left(\mathbf{u}_{1}-\mathbf{u}_{3}\right) \\
& -\left(\mathbf{u}_{2}-\mathbf{u}_{4}\right)^{T} \mathbf{R}_{2}\left(\mathbf{u}_{2}-\mathbf{u}_{4}\right)-\mathbf{u}_{4}^{T} \mathbf{S}^{T} \mathbf{u}_{2}+\mathbf{u}_{4}^{T} \mathbf{S}^{T} \mathbf{u}_{3} \\
& +\mathbf{u}_{2}^{T}\left[\mathbf{S}+\mathbf{S}^{T}-\mathbf{Q}_{3}\right] \mathbf{u}_{2}-\mathbf{u}_{2}^{T} \mathbf{S}^{T} \mathbf{u}_{3} \\
& -\left(\mathbf{u}_{2}-\mathbf{u}_{3}\right)^{T} \mathbf{R}_{2}\left(\mathbf{u}_{2}-\mathbf{u}_{3}\right)-\mathbf{u}_{2}^{T} \mathbf{S} \mathbf{u}_{4}-\mathbf{u}_{3}^{T} \mathbf{S} \mathbf{u}_{2} \\
& +\mathbf{u}_{3}^{T} \mathbf{S} \mathbf{u}_{4}-\mathbf{u}_{3}^{T} \mathbf{Q}_{1} \mathbf{u}_{3}-\mathbf{u}_{4}^{T} \mathbf{Q}_{2} \mathbf{u}_{4}+\mathbf{u}_{1}^{T} \mathbf{A}^{T} \mathbf{C} \mathbf{u}_{5} \\
& +\mathbf{u}_{5}^{T} \mathbf{C}^{T} \mathbf{A} \mathbf{u}_{1}+\mathbf{u}_{5}^{T} \mathbf{C}^{T} \overline{\mathbf{A}}_{d} \mathbf{u}_{2}+\mathbf{u}_{5}^{T} \mathbf{C}^{T} \mathbf{H}_{0} \mathbf{u}_{6} \\
& +\mathbf{u}_{5}^{T}\left[\mathbf{P}-\left(\mathbf{C}+\mathbf{C}^{T}\right)\right] \mathbf{u}_{5}+\mathbf{u}_{6}^{T} \mathbf{H}_{0}^{T} \mathbf{C} \mathbf{u}_{5} \\
& -\epsilon_{0} \mathbf{u}_{6}^{T} \mathbf{I} \mathbf{u}_{6}<\mathbf{0},
\end{aligned}
$$

where $\mathbf{u}_{i}(i=1,2, \ldots, 6)$ is the block entry matrix, for example, $\mathbf{u}_{3}=\left[\begin{array}{llllll}\mathbf{0} & \mathbf{0} & \mathbf{I} & \mathbf{0} & \mathbf{0} & \mathbf{0}\end{array}\right]$. Following the steps similar to
(34)-(36) along with the application of Schur's complement, (37) yields (20). This completes the proof of Theorem 6.

Remark 7. The numerical complexity of Theorem 6 is proportional to $L_{2} M_{2}^{3}$ [26] with $L_{2}$ (total row size of the LMIs in Theorem 6 ) $=2 n^{2}+11 n+2 p+2$ and $M_{2}$ (total number of scalar decision variables) $=6 n^{2}+n+2$. In the light of Remark 2 , it may be observed that Theorem 6 has smaller numerical complexity than Theorem 1 .

Next, for the situation where the system (1)-(8) is free of parameter uncertainties and saturation nonlinearities, Theorem 6 leads to the following corollary.

Corollary 8. Consider the system (1)-(8) in the absence of uncertainties and nonlinearities, that is, the system becomes

$$
\mathbf{x}(k+1)=\mathbf{A x}(k)+\mathbf{A}_{d} \mathbf{x}(k-d(k)) .
$$

Then, the system (38) with time-varying delay $d(k)$ satisfying (4) is globally asymptotically stable if there exist $n \times n$ positive definite symmetric matrices $\mathbf{P}, \mathbf{Q}_{i}(i=1,2,3), \mathbf{R}_{1}$, and $\mathbf{R}_{2}$ and a matrix $\mathbf{S}$ of appropriate dimensions such that the LMIs (19) and (39) are feasible:

$$
\begin{aligned}
& \overline{\mathbf{\Psi}}_{1}=\mathbf{e}_{1}^{T}\left[\mathbf{A}^{T} \mathbf{P A}-\mathbf{P}+\sum_{i=1}^{3} \mathbf{Q}_{i}+h_{12} \mathbf{Q}_{3}\right] \mathbf{e}_{1} \\
& +h_{12}^{2} \mathbf{e}_{1}^{T}\left(\mathbf{A}^{T} \mathbf{R}_{2} \mathbf{A}-\mathbf{A}^{T} \mathbf{R}_{2}-\mathbf{R}_{2} \mathbf{A}+\mathbf{R}_{2}\right) \mathbf{e}_{1} \\
& +h_{1}^{2} \mathbf{e}_{1}^{T}\left(\mathbf{R}_{1}+\mathbf{A}^{T} \mathbf{R}_{1} \mathbf{A}-\mathbf{A}^{T} \mathbf{R}_{1}-\mathbf{R}_{1} \mathbf{A}\right) \mathbf{e}_{1} \\
& +\mathbf{e}_{1}^{T}\left(\mathbf{A}^{T} \mathbf{P} \mathbf{A}_{d}+h_{1}^{2} \mathbf{A}^{T} \mathbf{R}_{1} \mathbf{A}_{d}-h_{1}^{2} \mathbf{R}_{1} \mathbf{A}_{d}\right. \\
& \left.+h_{12}^{2} \mathbf{A}^{T} \mathbf{R}_{2} \mathbf{A}_{d}-h_{12}^{2} \mathbf{R}_{2} \mathbf{A}_{d}\right) \mathbf{e}_{2} \\
& +\mathbf{e}_{2}^{T}\left(\mathbf{A}_{d}^{T} \mathbf{P A}+h_{1}^{2} \mathbf{A}_{d}^{T} \mathbf{R}_{1} \mathbf{A}-h_{1}^{2} \mathbf{A}_{d}^{T} \mathbf{R}_{1}\right. \\
& \left.+h_{12}^{2} \mathbf{A}_{d}^{T} \mathbf{R}_{2} \mathbf{A}-h_{12}^{2} \mathbf{A}_{d}^{T} \mathbf{R}_{2}\right) \mathbf{e}_{1} \\
& -\left(\mathbf{e}_{1}-\mathbf{e}_{3}\right)^{T} \mathbf{R}_{1}\left(\mathbf{e}_{1}-\mathbf{e}_{3}\right) \\
& -\left(\mathbf{e}_{2}-\mathbf{e}_{4}\right)^{T} \mathbf{R}_{2}\left(\mathbf{e}_{2}-\mathbf{e}_{4}\right) \\
& -\mathbf{e}_{4}^{T} \mathbf{S}^{T} \mathbf{e}_{2}+\mathbf{e}_{4}^{T} \mathbf{S}^{T} \mathbf{e}_{3}-\mathbf{e}_{2}^{T} \mathbf{S}^{T} \mathbf{e}_{3} \\
& +\mathbf{e}_{2}^{T}\left[\mathbf{S}+\mathbf{S}^{T}-\mathbf{Q}_{3}+\mathbf{A}_{d}^{T} \mathbf{P} \mathbf{A}_{d}\right. \\
& \left.+h_{1}^{2} \mathbf{A}_{d}^{T} \mathbf{R}_{1} \mathbf{A}_{d}+h_{12}^{2} \mathbf{A}_{d}^{T} \mathbf{R}_{2} \mathbf{A}_{d}\right] \mathbf{e}_{2} \\
& -\left(\mathbf{e}_{2}-\mathbf{e}_{3}\right)^{T} \mathbf{R}_{2}\left(\mathbf{e}_{2}-\mathbf{e}_{3}\right)-\mathbf{e}_{2}^{T} \mathbf{S e}_{4}-\mathbf{e}_{3}^{T} \mathbf{S e}_{2} \\
& +\mathbf{e}_{3}^{T} \mathbf{S} \mathbf{e}_{4}-\mathbf{e}_{3}^{T} \mathbf{Q}_{1} \mathbf{e}_{3}-\mathbf{e}_{4}^{T} \mathbf{Q}_{2} \mathbf{e}_{4}<\mathbf{0},
\end{aligned}
$$

where $\mathbf{e}_{i}(i=1,2,3,4)$ represents the block entry matrix, for example, $\mathbf{e}_{2}=\left[\begin{array}{llll}\mathbf{0} & \mathbf{I} & \mathbf{0} & \mathbf{0}\end{array}\right]$.

Proof. With $\mathbf{f}(\mathbf{y}(k))=\mathbf{y}(k)$ and $\Delta \mathbf{A}=\Delta \mathbf{A}_{d}=\mathbf{0}$ (which implies the absence of nonlinearities and uncertainties in the system (1)-(8)), (29) can be rearranged as

$$
\Delta V(\mathbf{x}(k)) \leq \boldsymbol{\xi}_{1}^{T}(k) \overline{\boldsymbol{\Psi}}_{1} \boldsymbol{\xi}_{1}(k),
$$


where

$$
\xi_{1}^{T}(k)=\left[\begin{array}{llll}
\mathbf{x}^{T}(k) & \mathbf{x}^{T}(k-d(k)) & \mathbf{x}^{T}\left(k-h_{1}\right) & \mathbf{x}^{T}\left(k-h_{2}\right)
\end{array}\right] .
$$

From (40), it is clear that $\Delta V(\mathbf{x}(k))<0$ if (19) and (39) hold true. This completes the proof of Corollary 8 .

Remark 9. It may be mentioned that a criterion for the global asymptotic stability of the system (38) is reported in [29] (see [29, Theorem 1]). It can be verified that Corollary 8 is the same as Theorem 1 of [29]. Thus, Theorem 1 of [29] is recovered from the presented approach as a special case. In other words, Theorem 6 may be considered as an extension of the delaydependent criterion given in Theorem 1 of [29] for timevarying delay systems to a model that includes, in addition, parameter uncertainty as well as saturation nonlinearities.

It may be observed that Theorem 6 has a free-weighting matrix S. Although the presence of these free-weighting matrices helps in obtaining reduced conservative results, but they contribute heavily towards the computational complexity.

Next, we present a criterion which does not include any free-weighting matrix.

Theorem 10. System (1)-(8) is globally asymptotically stable if there exist matrices $\mathbf{P}>\mathbf{0}, \mathbf{Q}_{i}>\mathbf{0}(i=1,2,3), \mathbf{R}_{1}>\mathbf{0}$, and $\mathbf{R}_{2}>\mathbf{0}$ and scalars $\epsilon_{0}>0, \epsilon_{1}>0, \bar{\epsilon}_{0}>0, \bar{\epsilon}_{1}>0, \alpha_{i j}>0$, and $\beta_{i j}>0(i, j=1,2, \ldots, n(i \neq j))$ such that $\boldsymbol{\Xi}_{1}<\mathbf{0}$ and $\boldsymbol{\Xi}_{2}<\mathbf{0}$ hold where

$$
\begin{aligned}
& \mathbf{\Xi}_{1}=\mathbf{v}_{1}^{T}\left[-\mathbf{P}+\sum_{i=1}^{3} \mathbf{Q}_{i}+h_{12} \mathbf{Q}_{3}+\epsilon_{0} \mathbf{E}_{0}^{T} \mathbf{E}_{0}\right] \mathbf{v}_{1} \\
& +\left(\mathbf{v}_{1}-\mathbf{v}_{5}\right)^{T}\left(h_{1}^{2} \mathbf{R}_{1}+h_{12}^{2} \mathbf{R}_{2}\right)\left(\mathbf{v}_{1}-\mathbf{v}_{5}\right) \\
& +\mathbf{v}_{2}^{T} \mathbf{A}_{d}^{T} \mathbf{C} \mathbf{v}_{5}-\left(\mathbf{v}_{1}-\mathbf{v}_{3}\right)^{T} \mathbf{R}_{1}\left(\mathbf{v}_{1}-\mathbf{v}_{3}\right) \\
& -\left(\mathbf{v}_{2}-\mathbf{v}_{3}\right)^{T} \mathbf{R}_{2}\left(\mathbf{v}_{2}-\mathbf{v}_{3}\right) \\
& +\mathbf{v}_{2}^{T}\left[-\mathbf{Q}_{3}+\epsilon_{1} \mathbf{E}_{1}^{T} \mathbf{E}_{1}\right] \mathbf{v}_{2} \\
& -\left(\mathbf{v}_{2}-\mathbf{v}_{4}\right)^{T} 3 \mathbf{R}_{2}\left(\mathbf{v}_{2}-\mathbf{v}_{4}\right) \\
& -\mathbf{v}_{3}^{T} \mathbf{Q}_{1} \mathbf{v}_{3}-\mathbf{v}_{4}^{T} \mathbf{Q}_{2} \mathbf{v}_{4}+\mathbf{v}_{1}^{T} \mathbf{A}^{T} \mathbf{C} \mathbf{v}_{5} \\
& +\mathbf{v}_{5}^{T} \mathbf{C}^{T} \mathbf{A} \mathbf{v}_{1}+\mathbf{v}_{5}^{T} \mathbf{C}^{T} \mathbf{A}_{d} \mathbf{v}_{2}+\mathbf{v}_{5}^{T} \mathbf{C}^{T} \mathbf{H}_{0} \mathbf{v}_{6} \\
& +\mathbf{v}_{5}^{T}\left[\mathbf{P}-\left(\mathbf{C}+\mathbf{C}^{T}\right)\right] \mathbf{v}_{5}+\mathbf{v}_{6}^{T} \mathbf{H}_{0}^{T} \mathbf{C} \mathbf{v}_{5} \\
& +\mathbf{v}_{5}^{T} \mathbf{C}^{T} \mathbf{H}_{1} \mathbf{v}_{7}+\mathbf{v}_{7}^{T} \mathbf{H}_{1}^{T} \mathbf{C} \mathbf{v}_{5}-\epsilon_{0} \mathbf{v}_{6}^{T} \mathbf{I} \mathbf{v}_{6} \\
& -\epsilon_{1} \mathbf{v}_{7}^{T} \mathbf{I} \mathbf{v}_{7} \text {, } \\
& \boldsymbol{\Xi}_{2}=\mathbf{v}_{1}^{T}\left[-\mathbf{P}+\sum_{i=1}^{3} \mathbf{Q}_{i}+h_{12} \mathbf{Q}_{3}+\bar{\epsilon}_{0} \mathbf{E}_{0}^{T} \mathbf{E}_{0}\right] \mathbf{v}_{1} \\
& +\left(\mathbf{v}_{1}-\mathbf{v}_{5}\right)^{T}\left(h_{1}^{2} \mathbf{R}_{1}+h_{12}^{2} \mathbf{R}_{2}\right)\left(\mathbf{v}_{1}-\mathbf{v}_{5}\right)
\end{aligned}
$$

$$
\begin{aligned}
& +\mathbf{v}_{2}^{T} \mathbf{A}_{d}^{T} \mathbf{C} \mathbf{v}_{5}-\left(\mathbf{v}_{1}-\mathbf{v}_{3}\right)^{T} \mathbf{R}_{1}\left(\mathbf{v}_{1}-\mathbf{v}_{3}\right) \\
& -\left(\mathbf{v}_{2}-\mathbf{v}_{3}\right)^{T} 3 \mathbf{R}_{2}\left(\mathbf{v}_{2}-\mathbf{v}_{3}\right) \\
& +\mathbf{v}_{2}^{T}\left[-\mathbf{Q}_{3}+\bar{\epsilon}_{1} \mathbf{E}_{1}^{T} \mathbf{E}_{1}\right] \mathbf{v}_{2} \\
& -\left(\mathbf{v}_{2}-\mathbf{v}_{4}\right)^{T} \mathbf{R}_{2}\left(\mathbf{v}_{2}-\mathbf{v}_{4}\right) \\
& -\mathbf{v}_{3}^{T} \mathbf{Q}_{1} \mathbf{v}_{3}-\mathbf{v}_{4}^{T} \mathbf{Q}_{2} \mathbf{v}_{4}+\mathbf{v}_{1}^{T} \mathbf{A}^{T} \mathbf{C} \mathbf{v}_{5} \\
& +\mathbf{v}_{5}^{T} \mathbf{C}^{T} \mathbf{A} \mathbf{v}_{1}+\mathbf{v}_{5}^{T} \mathbf{C}^{T} \mathbf{A}_{d} \mathbf{v}_{2}+\mathbf{v}_{5}^{T} \mathbf{C}^{T} \mathbf{H}_{0} \mathbf{v}_{6} \\
& +\mathbf{v}_{5}^{T}\left[\mathbf{P}-\left(\mathbf{C}+\mathbf{C}^{T}\right)\right] \mathbf{v}_{5}+\mathbf{v}_{6}^{T} \mathbf{H}_{0}^{T} \mathbf{C} \mathbf{v}_{5} \\
& +\mathbf{v}_{5}^{T} \mathbf{C}^{T} \mathbf{H}_{1} \mathbf{v}_{7}+\mathbf{v}_{7}^{T} \mathbf{H}_{1}^{T} \mathbf{C} \mathbf{v}_{5}-\bar{\epsilon}_{0} \mathbf{v}_{6}^{T} \mathbf{I} \mathbf{v}_{6} \\
& -\bar{\epsilon}_{1} \mathbf{v}_{7}^{T} \mathbf{I} \mathbf{v}_{7} .
\end{aligned}
$$

Proof. Recall the relation Equation (18) of [29]

$$
\begin{array}{r}
-h_{12} \sum_{i=k-h_{2}}^{k-h_{1}-1} \boldsymbol{\eta}^{T}(i) \mathbf{R}_{2} \boldsymbol{\eta}(i) \\
\leq \max \left\{-\gamma_{1}^{T}(k) \mathbf{R}_{2} \gamma_{1}(k)-3 \gamma_{2}^{T}(k) \mathbf{R}_{2} \gamma_{2}(k),\right. \\
\left.-3 \gamma_{1}^{T}(k) \mathbf{R}_{2} \gamma_{1}(k)-\gamma_{2}^{T}(k) \mathbf{R}_{2} \gamma_{2}(k)\right\},
\end{array}
$$

where $\gamma_{1}(k)$ and $\gamma_{2}(k)$ are given by (25) and (26), respectively. By choosing the Lyapunov functional (21) and using (27), (28), (30), and (43), we obtain

$$
\begin{aligned}
\Delta V(\mathbf{x}(k)) \leq \boldsymbol{\xi}^{T}(k) \boldsymbol{\Psi}_{0} \boldsymbol{\xi}(k) & \\
+\max \{- & \boldsymbol{\gamma}_{1}^{T}(k) \mathbf{R}_{2} \boldsymbol{\gamma}_{1}(k) \\
& -3 \boldsymbol{\gamma}_{2}^{T}(k) \mathbf{R}_{2} \gamma_{2}(k),-3 \gamma_{1}^{T}(k) \mathbf{R}_{2} \gamma_{1}(k) \\
& \left.-\boldsymbol{\gamma}_{2}^{T}(k) \mathbf{R}_{2} \boldsymbol{\gamma}_{2}(k)\right\}-\delta
\end{aligned}
$$

where

$$
\begin{aligned}
\boldsymbol{\Psi}_{0}= & \mathbf{e}_{1}^{T}\left[-\mathbf{P}+\sum_{i=1}^{3} \mathbf{Q}_{i}+h_{12} \mathbf{Q}_{3}\right] \mathbf{e}_{1} \\
& +\left(\mathbf{e}_{1}-\mathbf{e}_{5}\right)^{T}\left(h_{1}^{2} \mathbf{R}_{1}+h_{12}^{2} \mathbf{R}_{2}\right)\left(\mathbf{e}_{1}-\mathbf{e}_{5}\right) \\
& +\mathbf{e}_{2}^{T} \overline{\mathbf{A}}_{d}^{T} \mathbf{C} \mathbf{e}_{5}-\left(\mathbf{e}_{1}-\mathbf{e}_{3}\right)^{T} \mathbf{R}_{1}\left(\mathbf{e}_{1}-\mathbf{e}_{3}\right) \\
& -\mathbf{e}_{2}^{T} \mathbf{Q}_{3} \mathbf{e}_{2}-\mathbf{e}_{3}^{T} \mathbf{Q}_{1} \mathbf{e}_{3}-\mathbf{e}_{4}^{T} \mathbf{Q}_{2} \mathbf{e}_{4} \\
& +\mathbf{e}_{1}^{T} \overline{\mathbf{A}}^{T} \mathbf{C} \mathbf{e}_{5}+\mathbf{e}_{5}^{T} \mathbf{C}^{T} \overline{\mathbf{A}} \mathbf{e}_{1}+\mathbf{e}_{5}^{T} \mathbf{C}^{T} \overline{\mathbf{A}}_{d} \mathbf{e}_{2} \\
& +\mathbf{e}_{5}^{T}\left[\mathbf{P}-\left(\mathbf{C}+\mathbf{C}^{T}\right)\right] \mathbf{e}_{5}
\end{aligned}
$$


and $\delta$ is given by (30). Since $\delta$ is nonnegative $[12,14]$, $\Delta V(\mathbf{x}(k))<0$ if

$$
\begin{aligned}
& \Psi_{0}-\left(\mathbf{e}_{3}-\mathbf{e}_{2}\right)^{T} \mathbf{R}_{2}\left(\mathbf{e}_{3}-\mathbf{e}_{2}\right)-3\left(\mathbf{e}_{2}-\mathbf{e}_{4}\right)^{T} \mathbf{R}_{2}\left(\mathbf{e}_{2}-\mathbf{e}_{4}\right)<\mathbf{0}, \\
& \Psi_{0}-3\left(\mathbf{e}_{3}-\mathbf{e}_{2}\right)^{T} \mathbf{R}_{2}\left(\mathbf{e}_{3}-\mathbf{e}_{2}\right)-\left(\mathbf{e}_{2}-\mathbf{e}_{4}\right)^{T} \mathbf{R}_{2}\left(\mathbf{e}_{2}-\mathbf{e}_{4}\right)<\mathbf{0}
\end{aligned}
$$

hold. Here, $\mathbf{e}_{i}(i=1,2, \ldots, 5)$ is the block entry matrix, for example, $\mathbf{e}_{3}=\left[\begin{array}{lllll}\mathbf{0} & \mathbf{0} & \mathbf{I} & \mathbf{0} & \mathbf{0}\end{array}\right]$. Using the steps similar to (34)-(37), it can be shown that (46) and (47) yield the LMIs $\boldsymbol{\Xi}_{1}<\mathbf{0}$ and $\boldsymbol{\Xi}_{2}<\mathbf{0}$, respectively. This completes the proof of Theorem 10 .

Remark 11. The numerical complexity for Theorem 10 is given by $L_{3} M_{3}^{3}$ where $L_{3}=2 n^{2}+14 n+4 p+4$ and $M_{3}=5 n^{2}+n+$ 4. A comparison of the numerical complexity of Theorems 1 , 6 , and 10 is given in Table 1 with $p=1$. It may be observed that Theorem 10 provides smaller numerical complexity than Theorem 1. Further, from Table 1, it is clear that Theorem 10 has smaller numerical complexity than Theorem 6 for $n>2$.

As a consequence of Theorem 10, one can easily arrive at the following corollary.

Corollary 12. The system described by (38) is globally asymptotically stable if there exist matrices $\mathbf{P}>\mathbf{0}, \mathbf{Q}_{i}>\mathbf{0}(i=$ $1,2,3), \mathbf{R}_{1}>\mathbf{0}$, and $\mathbf{R}_{2}>\mathbf{0}$ such that

$$
\begin{aligned}
& \bar{\Psi}_{0}-\left(\mathbf{e}_{3}-\mathbf{e}_{2}\right)^{T} \mathbf{R}_{2}\left(\mathbf{e}_{3}-\mathbf{e}_{2}\right)-3\left(\mathbf{e}_{2}-\mathbf{e}_{4}\right)^{T} \mathbf{R}_{2}\left(\mathbf{e}_{2}-\mathbf{e}_{4}\right)<\mathbf{0}, \\
& \bar{\Psi}_{0}-3\left(\mathbf{e}_{3}-\mathbf{e}_{2}\right)^{T} \mathbf{R}_{2}\left(\mathbf{e}_{3}-\mathbf{e}_{2}\right)-\left(\mathbf{e}_{2}-\mathbf{e}_{4}\right)^{T} \mathbf{R}_{2}\left(\mathbf{e}_{2}-\mathbf{e}_{4}\right)<\mathbf{0},
\end{aligned}
$$

where

$$
\begin{aligned}
\bar{\Psi}_{0}= & \mathbf{e}_{1}^{T}\left[\mathbf{A}^{T} \mathbf{P} \mathbf{A}-\mathbf{P}+\sum_{i=1}^{3} \mathbf{Q}_{i}+h_{12} \mathbf{Q}_{3}\right] \mathbf{e}_{1} \\
& +h_{12}^{2} \mathbf{e}_{1}^{T}\left(\mathbf{A}^{T} \mathbf{R}_{2} \mathbf{A}-\mathbf{A}^{T} \mathbf{R}_{2}-\mathbf{R}_{2} \mathbf{A}+\mathbf{R}_{2}\right) \mathbf{e}_{1} \\
& +h_{1}^{2} \mathbf{e}_{1}^{T}\left(\mathbf{R}_{1}+\mathbf{A}^{T} \mathbf{R}_{1} \mathbf{A}-\mathbf{A}^{T} \mathbf{R}_{1}-\mathbf{R}_{1} \mathbf{A}\right) \mathbf{e}_{1} \\
& +\mathbf{e}_{1}^{T}\left(\mathbf{A}^{T} \mathbf{P} \mathbf{A}_{d}+h_{1}^{2} \mathbf{A}^{T} \mathbf{R}_{1} \mathbf{A}_{d}-h_{1}^{2} \mathbf{R}_{1} \mathbf{A}_{d}\right. \\
& \left.\quad+h_{12}^{2} \mathbf{A}^{T} \mathbf{R}_{2} \mathbf{A}_{d}-h_{12}^{2} \mathbf{R}_{2} \mathbf{A}_{d}\right) \mathbf{e}_{2} \\
& +\mathbf{e}_{2}^{T}\left(\mathbf{A}_{d}^{T} \mathbf{P} \mathbf{A}+h_{1}^{2} \mathbf{A}_{d}^{T} \mathbf{R}_{1} \mathbf{A}-h_{1}^{2} \mathbf{A}_{d}^{T} \mathbf{R}_{1}\right. \\
& \left.+h_{12}^{2} \mathbf{A}_{d}^{T} \mathbf{R}_{2} \mathbf{A}-h_{12}^{2} \mathbf{A}_{d}^{T} \mathbf{R}_{2}\right) \mathbf{e}_{1} \\
& -\left(\mathbf{e}_{1}-\mathbf{e}_{3}\right)^{T} \mathbf{R}_{1}\left(\mathbf{e}_{1}-\mathbf{e}_{3}\right) \\
& +\mathbf{e}_{2}^{T}\left[-\mathbf{Q}_{3}+\mathbf{A}_{d}^{T} \mathbf{P} \mathbf{A}_{d}\right. \\
& \left.+h_{1}^{2} \mathbf{A}_{d}^{T} \mathbf{R}_{1} \mathbf{A}_{d}+h_{12}^{2} \mathbf{A}_{d}^{T} \mathbf{R}_{2} \mathbf{A}_{d}\right] \mathbf{e}_{2} \\
& -\mathbf{e}_{3}^{T} \mathbf{Q}_{1} \mathbf{e}_{3}-\mathbf{e}_{4}^{T} \mathbf{Q}_{2} \mathbf{e}_{4} .
\end{aligned}
$$

Remark 13. One can easily verify that Corollary 12 is equivalent to Theorem 3 of [29]. Thus, Theorem 3 of [29] is recovered from the presented approach as special case. In other words, Theorem 10 may be treated as an extension to the criterion established in Theorem 3 of [29] for time-varying delay systems to a model that includes, in addition, normbounded uncertainty as well as saturation nonlinearities.

Remark 14. Theorems 6 and 10 are applicable for testing of global asymptotic stability of system (1)-(5) with timevarying uncertainties $\Delta \mathbf{A}=\mathbf{H}_{0} \mathbf{F}_{0}(k) \mathbf{E}_{0}$ and $\Delta \mathbf{A}_{d}=$ $\mathbf{H}_{1} \mathbf{F}_{1}(k) \mathbf{E}_{1}$ subject, of course, to $\mathbf{F}_{i}^{T}(k) \mathbf{F}_{i}(k) \leq \mathbf{I}(i=0,1)$.

Remark 15. The conditions given in Theorems 6 and 10 are in the form of LMIs and can be conveniently solved using MATLAB environment along with YALMIP 3.0 parser [42] and SeDuMi 1.21 solver [43].

\section{Numerical Example}

A comparative study of the presented criteria with the criterion in [18] is made to underline the usefulness of the presented results.

Example 1. Consider the system (1)-(8) with [18]

$$
\begin{array}{rlr}
\mathbf{A} & =\left[\begin{array}{cc}
0.8 & 0 \\
0.05 & 0.9
\end{array}\right], & \mathbf{A}_{d}=\left[\begin{array}{cc}
-0.1 & 0 \\
-0.2 & -0.1
\end{array}\right], \\
\mathbf{H}_{0}=\mathbf{H}_{1}=\left[\begin{array}{c}
0 \\
0.1
\end{array}\right], & \mathbf{E}_{0}=\left[\begin{array}{ll}
0.01 & 0
\end{array}\right], \quad \mathbf{E}_{1}=\left[\begin{array}{ll}
0 & 0.01
\end{array}\right] .
\end{array}
$$

In [18], the system under consideration was shown to be globally asymptotically stable via Theorem 1 for the delay range $2 \leq d(k) \leq 8$. It is checked that, for the same delay range, Theorem 6 succeeds to establish the global asymptotic stability of the present system. Theorem 10 also affirms the global asymptotic stability for this system over a delay range $2 \leq d(k) \leq 7$. From Table 2, it may be observed that for higher values of $h_{1}$, Theorem 10 succeeds in providing the same conservativeness as that of Theorems 1 and 6 . Further, as shown in Table 1, Theorem 6 is numerically less complex than Theorem 1 . However, Theorem 10 provides less computational burden than Theorems 1 and 6 (see Remarks 7 and 11).

By choosing an arbitrary initial condition, a plot of the state trajectories of the present system with $\mathbf{F}_{0}=\mathbf{F}_{1}=$ $\cos (180(k-1) / \pi)$ and $d(k)=2+\lfloor|6 \cos (180(k-1) / \pi)|\rceil$ is shown in Figure 1. The time-varying delay $d(k)$ and the uncertainty $\mathbf{F}_{0}=\mathbf{F}_{1}$ used in the simulation are shown in Figures 2 and 3, respectively. The simulation result in Figure 1 is consistent with the fact (which has been arrived at via Theorem 6) that the present system is globally asymptotically stable. Figure 4 depicts the plot of state trajectories of the nominal system (i.e., in the absence of uncertainties and delay) associated with the present example and supports the fact that the nominal system is globally asymptotically stable. 
TABLE 1: Comparison of numerical complexity.

\begin{tabular}{lccc}
\hline Methods & & Numerical complexity $L(M)^{3}$ & $n=4$ \\
& $n=2$ & $n=3$ & 2031995936 \\
Theorem 1 [18] & 17608192 & 273867968 & 84896640 \\
Theorem 6 & 746368 & 11295845 & 65421312 \\
Theorem 10 & 773344 & 9561344 & \\
\hline
\end{tabular}

TABLE 2: Upper delay bound $h_{2}$ for different $h_{1}$.

\begin{tabular}{lllllll}
\hline$h_{1}$ & 2 & 5 & 7 & 10 & 13 & \\
\hline Theorem 1 [18] & 8 & 8 & 9 & 11 & 14 & 20 \\
Theorem 6 & 8 & 8 & 9 & 11 & 14 & 20 \\
Theorem 10 & 7 & 8 & 9 & 11 & 14 & 20 \\
\hline
\end{tabular}

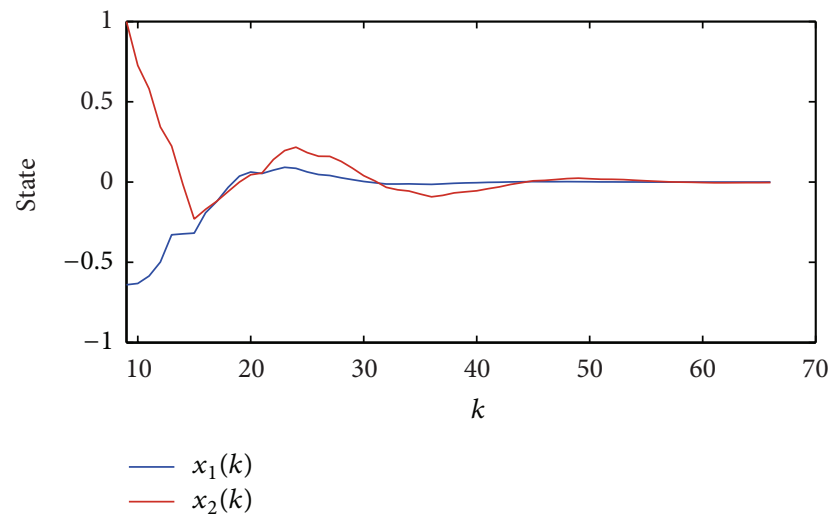

Figure 1: State response of the system in Example 1.

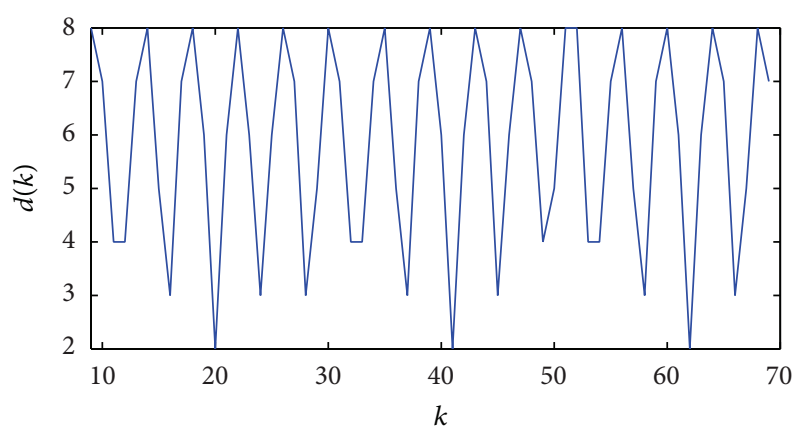

Figure 2: Time-varying delay $d(k)$ used in the simulation.

\section{Conclusions}

Two new delay-dependent stability criteria (Theorems 6 and 10) have been proposed for a class of uncertain discrete-time systems with time-varying delay in the presence of saturation nonlinearities. A numerical example has been considered for illustrating the effectiveness of the presented results. As compared to [18], the proposed criteria turn out to be numerically less complex. The presented approach can easily be extended to a class of nonlinear uncertain discrete-time systems with multiple time delays.

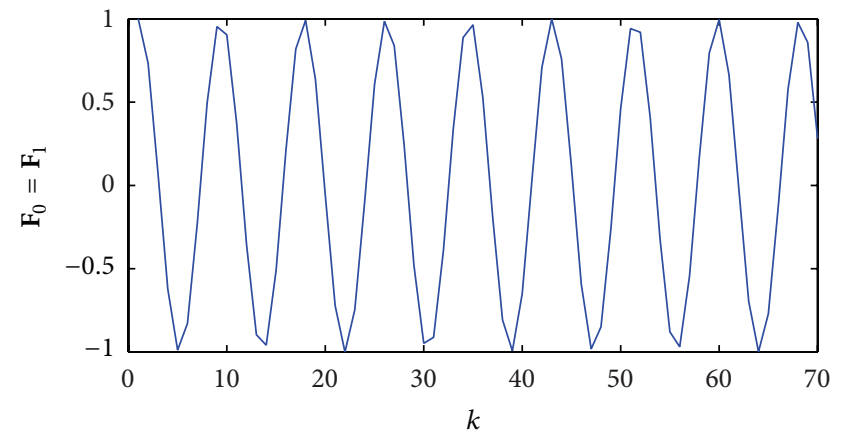

Figure 3: Norm-bounded uncertainty used in the simulation.

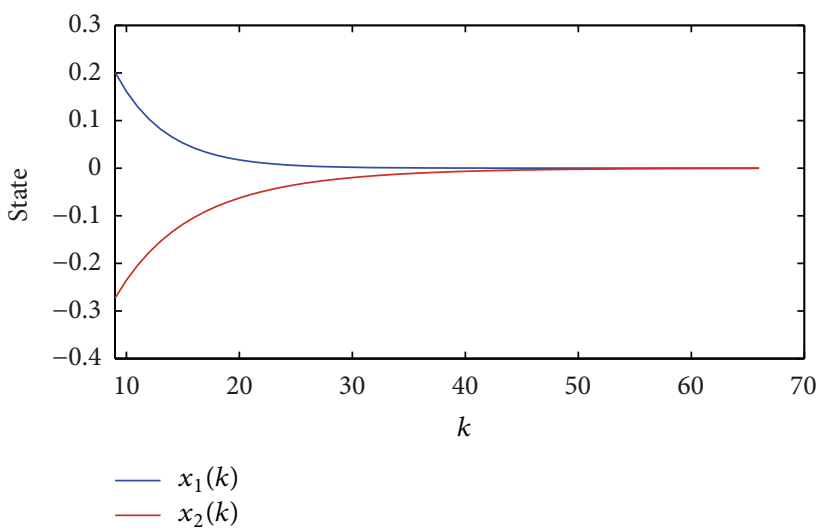

FIGURE 4: State response of the nominal system.

\section{Conflict of Interests}

The authors declare that there is no conflict of interests regarding the publication of this paper.

\section{References}

[1] T. A. C. M. Claasen, W. F. G. Mecklenbräuker, and J. B. H. Peek, "Effects of quantization and overflow in recursive digital filters," Institute of Electrical and Electronics Engineers. Transactions on 
Audio and Electroacoustics, vol. ASSP-24, no. 6, pp. 517-529, 1976.

[2] H. J. Butterweck, J. H. F. Ritzerfeld, and M. J. Werter, "Finite wordlength in digital filters-a review," EUT Report 88-E205, Eindhoven University of Technology, Eindhoven, The Netherlands, 1988.

[3] T. Ooba, "Stability of linear discrete dynamics employing state saturation arithmetic," Institute of Electrical and Electronics Engineers. Transactions on Automatic Control, vol. 48, no. 4, pp. 626-630, 2003.

[4] P. M. Ebert, J. E. Mazo, and M. G. Taylor, "Overflow oscillations in digital filters," Bell System Technical Journal, vol. 48, no. 9, pp. 2999-3020, 1969.

[5] W. L. Mills, C. T. Mullis, and R. A. Roberts, "Digital filter realizations without overflow oscillations," Institute of Electrical and Electronics Engineers. Transactions on Audio and Electroacoustics, vol. 26, no. 4, pp. 334-338, 1978.

[6] I. W. Sandberg, "The zero-input response of digital filters using saturation arithmetic," Institute of Electrical and Electronics Engineers. Transactions on Circuits and Systems, vol. 26, no. 11, pp. 911-915, 1979.

[7] J. H. F. Ritzerfeld, "A condition for the overflow stability of second-order digital filters that is satisfied by all scaled statespace structures using saturation," IEEE Transactions on Circuits and Systems, vol. 36, no. 8, pp. 1049-1057, 1989.

[8] T. Bose and M.-Q. Chen, "Overflow oscillations in state-space digital filters," IEEE Transactions on Circuits and Systems, vol. 38, no. 7, pp. 807-810, 1991.

[9] D. Liu and A. N. Michel, "Asymptotic stability of discretetime systems with saturation nonlinearities with applications to digital filters," IEEE Transactions on Circuits and Systems I, vol. 39, no. 10, pp. 798-807, 1992.

[10] H. Kar and V. Singh, "A new criterion for the overflow stability of second-order state-space digital filters using saturation arithmetic," IEEE Transactions on Circuits and Systems I, vol. 45, no. 3, pp. 311-313, 1998.

[11] H. Kar and V. Singh, "Elimination of overflow oscillations in fixed-point state-space digital filters with saturation arithmetic: an LMI approach," IEEE Transactions on Circuits and Systems II, vol. 51, no. 1, pp. 40-42, 2004.

[12] V. Singh, "Elimination of overflow oscillations in fixed-point state-space digital filters using saturation arithmetic," IEEE Transactions on Circuits and Systems, vol. 37, no. 6, pp. 814-818, 1990.

[13] V. Singh, "Elimination of overflow oscillations in fixed-point state-space digital filters using saturation arithmetic: an LMI approach," Digital Signal Processing, vol. 16, no. 1, pp. 45-51, 2006.

[14] H. Kar, "An LMI based criterion for the nonexistence of overflow oscillations in fixed-point state-space digital filters using saturation arithmetic," Digital Signal Processing, vol. 17, no. 3, pp. 685-689, 2007.

[15] S.-F. Chen, "Asymptotic stability of discrete-time systems with time-varying delay subject to saturation nonlinearities," Chaos, Solitons and Fractals, vol. 42, no. 2, pp. 1251-1257, 2009.

[16] V. K. R. Kandanvli and H. Kar, "Robust stability of discretetime state-delayed systems with saturation nonlinearities: linear matrix inequality approach," Signal Processing, vol. 89, no. 2, pp. 161-173, 2009.

[17] V. K. R. Kandanvli and H. Kar, "Delay-dependent LMI condition for global asymptotic stability of discrete-time uncertain state-delayed systems using quantization/overflow nonlinearities," International Journal of Robust and Nonlinear Control, vol. 21, no. 14, pp. 1611-1622, 2011.

[18] V. K. R. Kandanvli and H. Kar, "Delay-dependent stability criterion for discrete-time uncertain state-delayed systems employing saturation nonlinearities," Arabian Journal for Science and Engineering, vol. 38, no. 10, pp. 2911-2920, 2013.

[19] X. Ji, T. Liu, Y. Sun, and H. Su, "Stability analysis and controller synthesis for discrete linear time-delay systems with state saturation nonlinearities," International Journal of Systems Science, vol. 42, no. 3, pp. 397-406, 2011.

[20] M. S. Mahmoud, Robust Control and Filtering for Time-Delay Systems, vol. 5 of Control Engineering, Marcel Dekker, New York, NY, USA, 2000.

[21] S. Xu and J. Lam, "A survey of linear matrix inequality techniques in stability analysis of delay systems," International Journal of Systems Science, vol. 39, no. 12, pp. 1095-1113, 2008.

[22] S. Xu, J. Lam, and Y. Zou, "Improved conditions for delaydependent robust stability and stabilization of uncertain discrete time-delay systems," Asian Journal of Control, vol. 7, no. 3, pp. 344-348, 2005.

[23] H. Huang and G. Feng, "Improved approach to delaydependent stability analysis of discrete-time systems with timevarying delay," IET Control Theory \& Applications, vol. 4, no. 10, pp. 2152-2159, 2010.

[24] H. Shao and Q.-L. Han, "New stability criteria for linear discrete-time systems with interval-like time-varying delays," Institute of Electrical and Electronics Engineers. Transactions on Automatic Control, vol. 56, no. 3, pp. 619-625, 2011.

[25] W.-H. Chen, Z.-H. Guan, and X. Lu, "Delay-dependent guaranteed cost control for uncertain discrete-time systems with delay," IEE Proceedings Control Theory and Applications, vol. 150, no. 4, pp. 412-416, 2003.

[26] Q.-L. Han, "Improved stability criteria and controller design for linear neutral systems," Automatica, vol. 45, no. 8, pp. 1948-1952, 2009.

[27] Y. Wu, Y. Wu, and Y. Chen, "Mean square exponential stability of uncertain stochastic neural networks with time-varying delay," Neurocomputing, vol. 72, no. 10-12, pp. 2379-2384, 2009.

[28] X. Jiang, Q.-L. Han, and X. Yu, "Stability criteria for linear discrete-time systems with interval-like time-varying delay," in Proceedings of the American Control Conference (ACC '05), pp. 2817-2822, Portland, Ore, USA, June 2005.

[29] J. Liu and J. Zhang, "Note on stability of discrete-time timevarying delay systems," IET Control Theory \& Applications, vol. 6, no. 2, pp. 335-339, 2012.

[30] X. Meng, J. Lam, B. Du, and H. Gao, "A delay-partitioning approach to the stability analysis of discrete-time systems," Automatica, vol. 46, no. 3, pp. 610-614, 2010.

[31] P. Kokil, H. Kar, and V. K. R. Kandanvli, "Stability analysis of linear discrete-time systems with interval delay: a delaypartitioning approach," ISRN Applied Mathematics, vol. 2011, Article ID 624127, 10 pages, 2011.

[32] V. K. R. Kandanvli and H. Kar, "A delay-dependent approach to stability of uncertain discrete-time statedelayed systems with generalized over flow nonlinearities," ISRN Computational Mathematics, vol. 2012, Article ID 171606, 8 pages, 2012.

[33] Y. He, M. Wu, G.-P. Liu, and J.-H. She, "Output feedback stabilization for a discrete-time system with a time-varying delay," Institute of Electrical and Electronics Engineers. Transactions on Automatic Control, vol. 53, no. 10, pp. 2372-2377, 2008. 
[34] B. Zhang, S. Xu, and Y. Zou, "Improved stability criterion and its applications in delayed controller design for discrete-time systems," Automatica, vol. 44, no. 11, pp. 2963-2967, 2008.

[35] H. Gao and T. Chen, "New results on stability of discrete-time systems with time-varying state delay," Institute of Electrical and Electronics Engineers. Transactions on Automatic Control, vol. 52, no. 2, pp. 328-334, 2007.

[36] H. Gao, J. Lam, C. Wang, and Y. Wang, "Delay-dependent output-feedback stabilisation of discrete-time systems with time-varying state delay," IEE Proceedings Control Theory and Applications, vol. 151, no. 6, pp. 691-698, 2004.

[37] O. M. Kwon, M. J. Park, J. H. Park, S. M. Lee, and E. J. Cha, "Improved delay-dependent stability criteria for discrete-time systems with time-varying delays," Circuits, Systems, and Signal Processing, vol. 32, no. 4, pp. 1949-1962, 2013.

[38] L. Bakule, J. Rodellar, and J. M. Rossell, "Robust overlapping guaranteed cost control of uncertain state-delay discrete-time systems," Institute of Electrical and Electronics Engineers. Transactions on Automatic Control, vol. 51, no. 12, pp. 1943-1950, 2006.

[39] P. Park, J. W. Ko, and C. Jeong, "Reciprocally convex approach to stability of systems with time-varying delays," Automatica, vol. 47, no. 1, pp. 235-238, 2011.

[40] L. Xie, M. Fu, and C. E. de Souza, " $H_{\infty}$-control and quadratic stabilization of systems with parameter uncertainty via output feedback," Institute of Electrical and Electronics Engineers. Transactions on Automatic Control, vol. 37, no. 8, pp. 1253-1256, 1992.

[41] S. Boyd, L. El Ghaoui, E. Feron, and V. Balakrishnan, Linear Matrix Inequalities in System and Control Theory, vol. 15 of SIAM Studies in Applied Mathematics, Society for Industrial and Applied Mathematics (SIAM), Philadelphia, Pa, USA, 1994.

[42] J. Löfberg, "YALMIP: a toolbox for modeling and optimization in MATLAB," in Proceedings of the IEEE International Symposium on Computer Aided Control System Design, pp. 284-289, Taipei, Taiwan, September 2004.

[43] J. F. Sturm, "Using SeDuMi 1.02, a MATLAB toolbox for optimization over symmetric cones," Optimization Methods and Software, vol. 11/12, no. 1-4, pp. 625-653, 1999. 


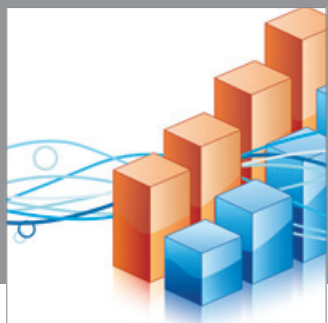

Advances in

Operations Research

mansans

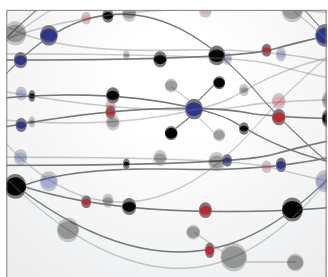

The Scientific World Journal
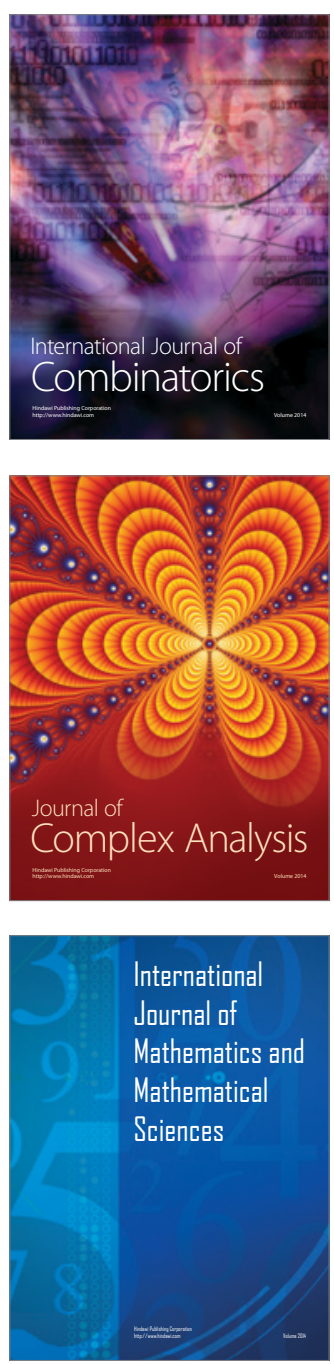
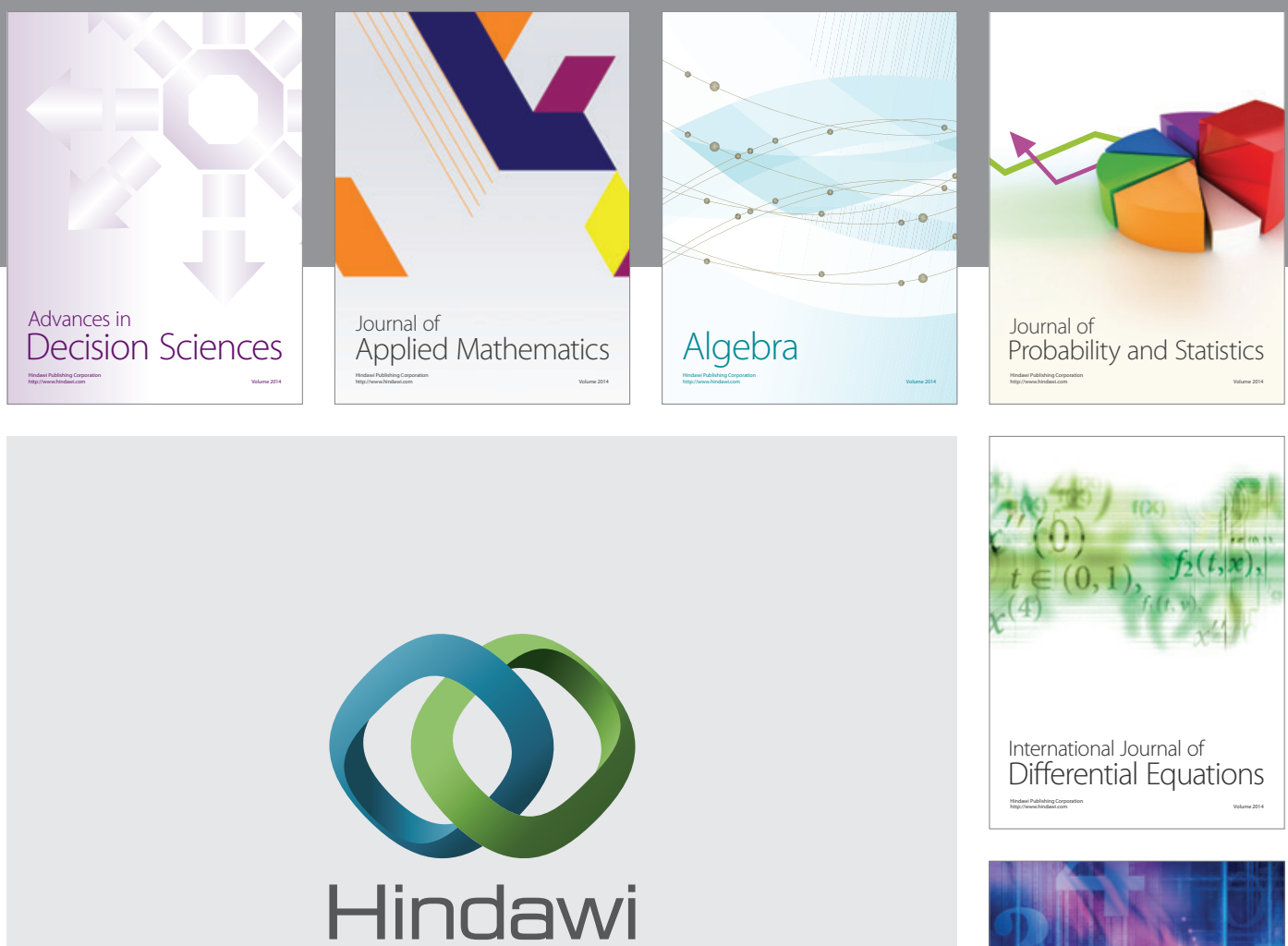

Submit your manuscripts at http://www.hindawi.com
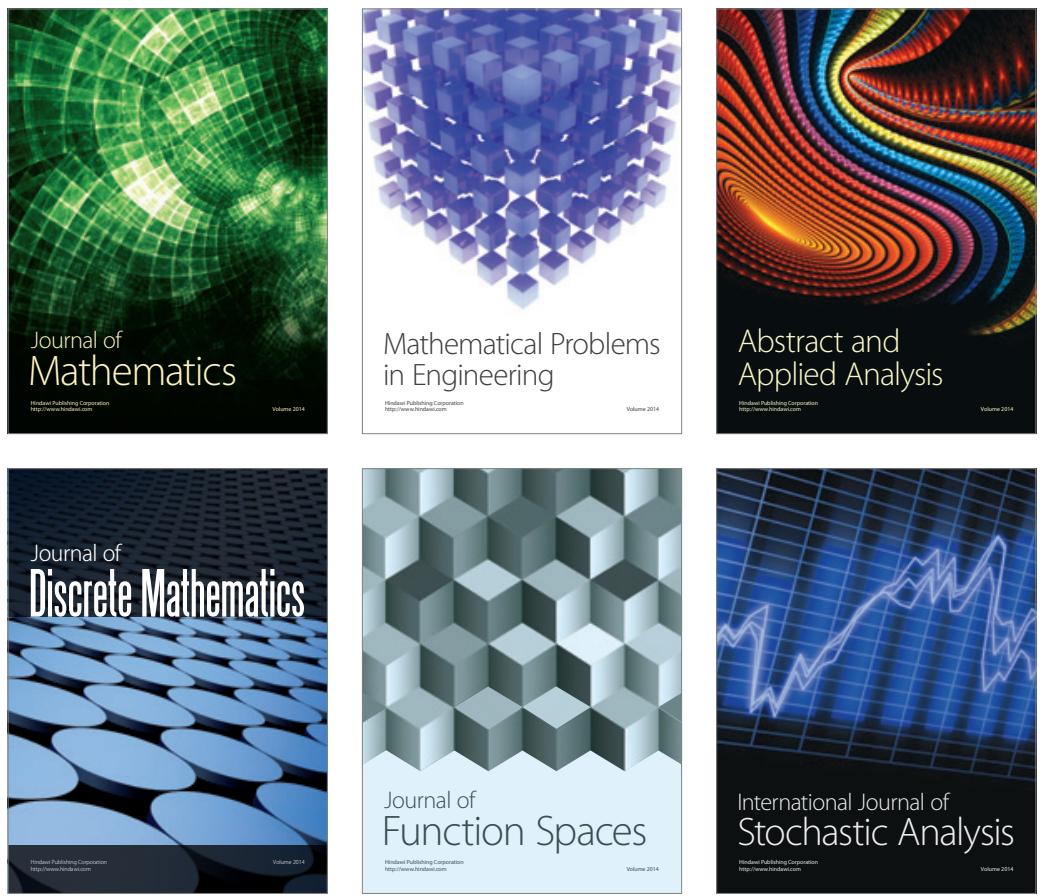

Journal of

Function Spaces

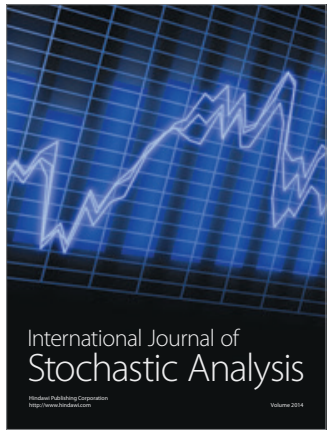

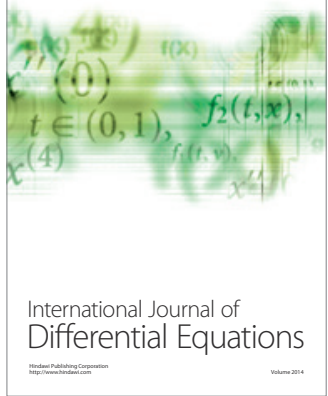
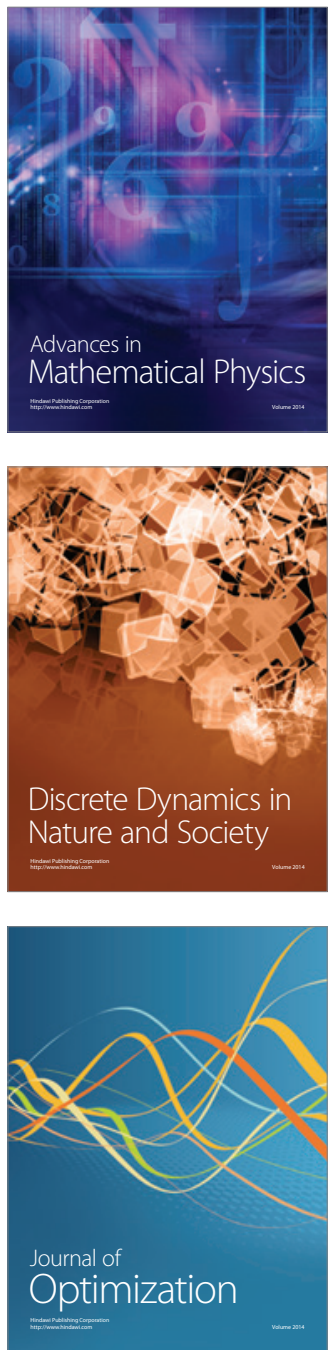